\title{
Studies on Synthesis of Some New Chalcone and Pyrimidines and their Antibacterial Activity
}

\author{
KETAN MISTRY and K. R. DESAI* \\ Department of Chemistry \\ Veer Narmad South Gujarat University \\ Surat - 395007 (India)
}

Received 15 February 2005; Accepted 25 March 2005

\begin{abstract}
Pyrimidine-2-one derivatives, 2-[2-\{1'-(p-nitrophenyl)-6'-(substitutedphenyl)-pyrimidine-2'-one-4'-yl \}-hydrazinomethyl]-3-(p-methoxy phenyl)-quinazoline-4(3H)-one [4a-j] have been synthesised by the condensation of p-nitro phenylurea and various chalcones, 2-(substituted phenylchalconylhydrazinomethyl)-3(p-methoxyphenyl)-quinazoline-4(3H)-one [3a-j] in the presence of catalytic amount of conc. HCl. And this series of chalcones have been synthesised by the reaction of 2 acetylhydrazinomethyl-3-(substitutedphenyl)-quinazoline-4 $(3 \mathrm{H})$-one [2] with different substituted aldehydes in presence of a $2 \% \mathrm{NaOH}$ solution. These products have been characterized by means of elemental analysis, IR and NMR spectrul studies and tested for antibacterial activities against Staphylococcus aureus, Bacillus subtillis, Escherichia coli and Salmonella typhi.
\end{abstract}

Key words: Synthesis, Quinazoline, Chalcone, Pyrimidine, Antibacterial activity.

\section{Introduction}

The chemistry and pharmacology of quinazolinone have been of great interest to medicinal chemistry because quinazolinone derivatives possessed various biological activities, such as anti-inflammatory ${ }^{1}$, antibacterial ${ }^{2}, \mathrm{CVS}^{3}$ and anticonvulsant ${ }^{4}$. Chalcones and their derivatives possess some interesting biological properties such as antibacterial ${ }^{5}$, antifungal ${ }^{6}$, insecticidal ${ }^{7}$, analgesic, anti-inflammatory and ulcerogenic ${ }^{8}$ etc.

Pyrimidines and their derivatives are considered to be important for drugs and agricultural chemicals. Pyrimidine derivatives possess several interesting biological activities such as antimicrobial $^{9}$, antitumour ${ }^{10}$ and antifungal activities ${ }^{11}$. Many Pyrimidine derivatives are used for thyroid drugs and leukemia. The starting compound 3-(p-methoxy phenyl)-2-hydrazinomethylquinazoline-4(3H)-one [1] can be synthesised according to literature method ${ }^{12}$. 


\section{Experimental}

All the melting points were determined in open capillaries and are uncorrected. The purity of compounds was checked by TLC on silica gel ' $G$ ' coated glass plates. IR spectra were recorded with $\mathrm{KBr}$ on Shimadzu FT-IR 8300 spectrophotometer, ${ }^{1} \mathrm{H}$ NMR spectra on a Brucker DRX - 300 in $\mathrm{CDCl}_{3}$ at $200 \mathrm{MHz}$ using TMS as an internal standard.<smiles>CC(=O)CCC(C)=O</smiles>

[1]<smiles>[R]c1ccc(C2CC(N)=NC(=O)N2c2ccc([N+](=O)[O-])cc2)cc1</smiles>

Scheme

Preparation of 2-acetylhydrazinemethyl-3-(p-methoxy phenyl)-quinazoline-4(3H)-one. [2]

In a $250 \mathrm{ml}$ conical flask take 2-hydrazino methyl-3-(p-methoxy phenyl)-quinazoline-4(3H)-one [1] ( $0.01 \mathrm{~mole})$ in chloroform (dry, $50 \mathrm{ml})$, acetyl chloride $(0.01 \mathrm{~mole})$ was added drop wise with constant stirring at $0-5{ }^{\circ} \mathrm{C}$. The reaction mixture was stirred for 8 hours, the excess solvent was distilled off and the separated solid thus obtained was poured into ice water. The resulting solid was filtered, washed with water, dried and recrystallised from ether. m.p. $-156{ }^{\circ} \mathrm{C}$. yield $-60 \%$. 
Preparation of 2-(4-chloro phenylchalconylhydrazinomethyl)-3-(p-methoxy phenyl)-quinazoline4(3H)-one. [3j]

In a R.B.F. take 2-acetylhydrazinemethyl-3-(p-methoxy phenyl)-quinazoline-4(3H)-one [2] (0.01 mole) in methanol. Then add p-chloro benzaldehyde $(0.01$ mole $)$ and $5 \mathrm{ml}$ of $2 \% \mathrm{NaOH}$ solution. The reaction mixture was refluxed for 5 hours and then poured into ice water. The resulting mass thus separated was filtered and washed with water, dried and recrystallised from ethanol.

IR (KBr): $3362(-\mathrm{NH}), 1687$ (-NHCO), $1637(-\mathrm{CH}=\mathrm{CH}), 1675(-\mathrm{C}=\mathrm{N}), 1706(-\mathrm{C}=\mathrm{O}), 850$ (-C-Cl); ${ }^{1}$ H NMR: $\delta 4.66\left(\mathrm{~s}, 2 \mathrm{H},-\mathrm{CH}_{2}\right), 5.46(\mathrm{~s}, 1 \mathrm{H},-\mathrm{NH}), 5.98(\mathrm{~d}, 1 \mathrm{H},-\mathrm{COCH}), 6.58(\mathrm{~s}, 1 \mathrm{H},-\mathrm{NHCO}), 7.18-$ $7.64(\mathrm{~m}, 12 \mathrm{H}, \mathrm{Ar}-\mathrm{H}), 8.40(\mathrm{~d}, 1 \mathrm{H},=\mathrm{CH}-\mathrm{Ar}), 3.42\left(\mathrm{~s}, 3 \mathrm{H}, \mathrm{Ar}-\mathrm{OCH}_{3}\right)$.

Other chalcone derivatives of this series were prepared in a similar manner. Data of the various chalcone derivatives are given in Table-I.

Preparation of 2-[2-\{1'-(p-nitrophenyl)-6'-(p-chloro phenyl)-pyrimidine-2'-one-4'-yl\}hydrazinomethyl]-3-(p-methoxy phenyl)-quinazoline-4(3H)-one. [4j]

In a R.B.F. take 2-(4-chloro phenylchalconylhydrazino methyl)-3-(p-methoxy phenyl)-quinazoline$4(3 \mathrm{H})$-one [3j] $(0.01 \mathrm{~mole})$ in methanol. Then add p-Nitro phenylurea $(0.01 \mathrm{~mole})$ and catalytic amount of conc. $\mathrm{HCl}$. The reaction mixture was refluxed for about 6 hours and poured into ice water. The solid product obtained was filtered and washed with water, recrystallised from absolute alcohol.

IR (KBr): $3360(-\mathrm{NH}), 1675(-\mathrm{C}=\mathrm{N}), 1706(-\mathrm{C}=\mathrm{O}), 850(-\mathrm{C}-\mathrm{Cl}), 1512\left(-\mathrm{NO}_{2}\right)$;

${ }^{1}$ H NMR: $\delta 4.61\left(\mathrm{~s}, 4 \mathrm{H},-\mathrm{CH}_{2}\right), 5.41(\mathrm{~s}, 2 \mathrm{H},-\mathrm{NH}), 7.18-7.64(\mathrm{~m}, 16 \mathrm{H}, \mathrm{Ar}-\mathrm{H}), 3.45\left(\mathrm{~s}, 3 \mathrm{H}, \mathrm{Ar}_{-} \mathrm{OCH}_{3}\right)$.

Other pyrimidine-2-one derivatives of this series were prepared in a similar manner. Data of the various pyrimidine-2-one derivatives are given in Table-II.

Table - I Analytical Data of Chalcone Derivatives

\begin{tabular}{|c|c|c|c|c|c|c|c|}
\hline \multirow[t]{2}{*}{ No. } & \multirow[t]{2}{*}{$\mathrm{R}$} & \multirow{2}{*}{$\begin{array}{l}\text { M.F. } \\
\text { (M.W.) }\end{array}$} & \multirow{2}{*}{$\begin{array}{l}\text { Yield } \\
(\%)\end{array}$} & \multirow{2}{*}{$\begin{array}{l}\text { M.P. } \\
\left({ }^{0} \mathrm{C}\right)\end{array}$} & \multicolumn{3}{|c|}{$\begin{array}{c}\% \text { Analysis } \\
\text { Found (Calc.) }\end{array}$} \\
\hline & & & & & $\mathrm{C}$ & $\mathrm{H}$ & $\mathrm{N}$ \\
\hline $3 a$ & $\begin{array}{c}3^{\prime}-\mathrm{OCH}_{3}, \\
\text { 4'-OH }\end{array}$ & $\begin{array}{c}\mathrm{C}_{26} \mathrm{H}_{24} \mathrm{~N}_{4} \mathrm{O}_{5} \\
(472.49)\end{array}$ & 63 & 125 & $\begin{array}{c}66.11 \\
(66.09)\end{array}$ & $\begin{array}{c}5.15 \\
(5.12)\end{array}$ & $\begin{array}{r}11.84 \\
(11.86)\end{array}$ \\
\hline $3 b$ & $4^{\prime}-\mathrm{CH}_{3}$ & $\begin{array}{c}\mathrm{C}_{26} \mathrm{H}_{24} \mathrm{~N}_{4} \mathrm{O}_{3} \\
(440.49)\end{array}$ & 60 & 105 & $\begin{array}{c}70.92 \\
(70.89)\end{array}$ & $\begin{array}{c}5.46 \\
(5.49)\end{array}$ & $\begin{array}{r}12.76 \\
(12.72)\end{array}$ \\
\hline $3 c$ & $2^{\prime}-\mathrm{OH}$ & $\begin{array}{c}\mathrm{C}_{25} \mathrm{H}_{22} \mathrm{~N}_{4} \mathrm{O}_{4} \\
(442.46)\end{array}$ & 63 & 114 & $\begin{array}{c}67.89 \\
(67.86)\end{array}$ & $\begin{array}{c}5.03 \\
(5.01)\end{array}$ & $\begin{array}{c}12.65 \\
(12.66)\end{array}$ \\
\hline $3 d$ & $4^{\prime}-\mathrm{OCH}_{3}$ & $\begin{array}{c}\mathrm{C}_{26} \mathrm{H}_{24} \mathrm{~N}_{4} \mathrm{O}_{4} \\
(456.49)\end{array}$ & 65 & 122 & $\begin{array}{c}68.40 \\
(68.41)\end{array}$ & $\begin{array}{c}5.32 \\
(5.30)\end{array}$ & $\begin{array}{r}12.29 \\
(12.27)\end{array}$ \\
\hline $3 e$ & $3^{\prime}-\mathrm{NO}_{2}$ & $\begin{array}{c}\mathrm{C}_{25} \mathrm{H}_{21} \mathrm{~N}_{5} \mathrm{O}_{5} \\
(471.46)\end{array}$ & 62 & 119 & $\begin{array}{c}63.68 \\
(63.69)\end{array}$ & $\begin{array}{c}4.46 \\
(4.49)\end{array}$ & $\begin{array}{c}14.88 \\
(14.85)\end{array}$ \\
\hline $3 \mathrm{f}$ & $4^{\prime}-\mathrm{N}\left(\mathrm{CH}_{3}\right)_{2}$ & $\begin{array}{c}\mathrm{C}_{27} \mathrm{H}_{27} \mathrm{~N}_{5} \mathrm{O}_{3} \\
(469.53)\end{array}$ & 65 & 128 & $\begin{array}{c}69.05 \\
(69.07)\end{array}$ & $\begin{array}{c}5.82 \\
(5.80)\end{array}$ & $\begin{array}{r}14.95 \\
(14.92)\end{array}$ \\
\hline $3 g$ & $2^{\prime}-\mathrm{NO}_{2}$ & $\begin{array}{c}\mathrm{C}_{25} \mathrm{H}_{21} \mathrm{~N}_{5} \mathrm{O}_{5} \\
(471.46)\end{array}$ & 60 & 113 & $\begin{array}{c}63.66 \\
(63.69)\end{array}$ & $\begin{array}{c}4.45 \\
(4.49)\end{array}$ & $\begin{array}{r}14.88 \\
(14.85)\end{array}$ \\
\hline $3 \mathrm{~h}$ & $4^{\prime}-\mathrm{F}$ & $\begin{array}{c}\mathrm{C}_{25} \mathrm{H}_{21} \mathrm{~N}_{4} \mathrm{O}_{3} \mathrm{~F} \\
(444.45)\end{array}$ & 62 & 130 & $\begin{array}{c}67.52 \\
(67.56)\end{array}$ & $\begin{array}{c}4.79 \\
(4.76)\end{array}$ & $\begin{array}{r}12.64 \\
(12.61)\end{array}$ \\
\hline $3 \mathrm{i}$ & $2^{\prime}-\mathrm{OCH}_{3}$ & $\begin{array}{c}\mathrm{C}_{26} \mathrm{H}_{24} \mathrm{~N}_{4} \mathrm{O}_{4} \\
(456.49)\end{array}$ & 65 & 117 & $\begin{array}{c}68.45 \\
(68.41)\end{array}$ & $\begin{array}{c}5.26 \\
(5.30)\end{array}$ & $\begin{array}{c}12.26 \\
(12.27)\end{array}$ \\
\hline $3 j$ & $4^{\prime}-\mathrm{Cl}$ & $\begin{array}{c}\mathrm{C}_{25} \mathrm{H}_{21} \mathrm{~N}_{4} \mathrm{O}_{3} \mathrm{Cl} \\
(460.91)\end{array}$ & 55 & 112 & $\begin{array}{c}65.13 \\
(65.15)\end{array}$ & $\begin{array}{c}4.57 \\
(4.59)\end{array}$ & $\begin{array}{c}12.19 \\
(12.16)\end{array}$ \\
\hline
\end{tabular}


Table - II _Analytical Data of Pyrimidine Derivatives

\begin{tabular}{|c|c|c|c|c|c|c|c|}
\hline \multirow[t]{2}{*}{ No. } & \multirow[t]{2}{*}{$\mathrm{R}$} & \multirow{2}{*}{$\begin{array}{l}\text { M.F. } \\
\text { (M.W.) }\end{array}$} & \multirow{2}{*}{$\begin{array}{l}\text { Yield } \\
(\%)\end{array}$} & \multirow{2}{*}{$\begin{array}{l}\text { M.P. } \\
\left({ }^{0} \mathrm{C}\right)\end{array}$} & \multicolumn{3}{|c|}{$\begin{array}{c}\% \text { Analysis } \\
\text { Found (Calc.) }\end{array}$} \\
\hline & & & & & $\mathrm{C}$ & $\mathrm{H}$ & $\mathrm{N}$ \\
\hline $4 a$ & $\begin{array}{c}\text { 3'- }-\mathrm{OCH}_{3}, \\
\text { 4'-OH }\end{array}$ & $\begin{array}{c}\mathrm{C}_{33} \mathrm{H}_{29} \mathrm{~N}_{7} \mathrm{O}_{7} \\
(635.62)\end{array}$ & 52 & 140 & $\begin{array}{c}62.38 \\
(62.36)\end{array}$ & $\begin{array}{c}4.62 \\
(4.60)\end{array}$ & $\begin{array}{c}15.41 \\
(15.43)\end{array}$ \\
\hline $4 b$ & $4^{\prime}-\mathrm{CH}_{3}$ & $\begin{array}{c}\mathrm{C}_{33} \mathrm{H}_{29} \mathrm{~N}_{7} \mathrm{O}_{5} \\
(603.62)\end{array}$ & 50 & 123 & $\begin{array}{c}65.69 \\
(65.66)\end{array}$ & $\begin{array}{c}4.87 \\
(4.84)\end{array}$ & $\begin{array}{c}16.21 \\
(16.24)\end{array}$ \\
\hline $4 c$ & $2^{\prime}-\mathrm{OH}$ & $\begin{array}{c}\mathrm{C}_{32} \mathrm{H}_{27} \mathrm{~N}_{7} \mathrm{O}_{6} \\
(605.60)\end{array}$ & 56 & 131 & $\begin{array}{c}63.43 \\
(63.46)\end{array}$ & $\begin{array}{c}4.45 \\
(4.49)\end{array}$ & $\begin{array}{l}16.15 \\
(16.19)\end{array}$ \\
\hline $4 d$ & $4^{\prime}-\mathrm{OCH}_{3}$ & $\begin{array}{c}\mathrm{C}_{33} \mathrm{H}_{29} \mathrm{~N}_{7} \mathrm{O}_{6} \\
(619.62)\end{array}$ & 60 & 138 & $\begin{array}{c}63.99 \\
(63.97)\end{array}$ & $\begin{array}{c}4.74 \\
(4.72)\end{array}$ & $\begin{array}{c}15.81 \\
(15.82)\end{array}$ \\
\hline $4 e$ & $3^{\prime}-\mathrm{NO}_{2}$ & $\begin{array}{c}\mathrm{C}_{32} \mathrm{H}_{26} \mathrm{~N}_{8} \mathrm{O}_{7} \\
(634.59)\end{array}$ & 58 & 135 & $\begin{array}{l}60.53 \\
(60.56)\end{array}$ & $\begin{array}{c}4.11 \\
(4.13)\end{array}$ & $\begin{array}{c}17.63 \\
(17.66)\end{array}$ \\
\hline $4 \mathrm{f}$ & $4^{\prime}-\mathrm{N}\left(\mathrm{CH}_{3}\right)_{2}$ & $\begin{array}{c}\mathrm{C}_{34} \mathrm{H}_{32} \mathrm{~N}_{8} \mathrm{O}_{5} \\
(632.66)\end{array}$ & 59 & 141 & $\begin{array}{c}64.57 \\
(64.55)\end{array}$ & $\begin{array}{c}5.13 \\
(5.10)\end{array}$ & $\begin{array}{l}17.75 \\
(17.71)\end{array}$ \\
\hline $4 \mathrm{~g}$ & $2^{\prime}-\mathrm{NO}_{2}$ & $\begin{array}{c}\mathrm{C}_{32} \mathrm{H}_{26} \mathrm{~N}_{8} \mathrm{O}_{7} \\
(634.59)\end{array}$ & 60 & 132 & $\begin{array}{c}60.52 \\
(60.56)\end{array}$ & $\begin{array}{c}4.12 \\
(4.13)\end{array}$ & $\begin{array}{l}17.65 \\
(17.66)\end{array}$ \\
\hline $4 \mathrm{~h}$ & $4^{\prime}-\mathrm{F}$ & $\begin{array}{c}\mathrm{C}_{32} \mathrm{H}_{26} \mathrm{~N}_{7} \mathrm{O}_{5} \mathrm{~F} \\
(607.59)\end{array}$ & 58 & 145 & $\begin{array}{c}63.29 \\
(63.26)\end{array}$ & $\begin{array}{c}4.30 \\
(4.31)\end{array}$ & $\begin{array}{l}16.11 \\
(16.14)\end{array}$ \\
\hline $4 \mathrm{i}$ & $2^{\prime}-\mathrm{OCH}_{3}$ & $\begin{array}{c}\mathrm{C}_{33} \mathrm{H}_{29} \mathrm{~N}_{7} \mathrm{O}_{6} \\
(619.62)\end{array}$ & 57 & 142 & $\begin{array}{c}63.95 \\
(63.97)\end{array}$ & $\begin{array}{c}4.70 \\
(4.72)\end{array}$ & $\begin{array}{c}15.81 \\
(15.82)\end{array}$ \\
\hline $4 j$ & $4^{\prime}-\mathrm{Cl}$ & $\begin{array}{c}\mathrm{C}_{32} \mathrm{H}_{26} \mathrm{~N}_{7} \mathrm{O}_{5} \mathrm{Cl} \\
(624.04)\end{array}$ & 51 & 129 & $\begin{array}{c}61.58 \\
(61.59)\end{array}$ & $\begin{array}{c}4.23 \\
(4.20)\end{array}$ & $\begin{array}{c}15.70 \\
(15.71)\end{array}$ \\
\hline
\end{tabular}

Antimicrobial activity

The synthesized compounds were tested for their antibacterial activity by measuring the zone of inhibition on agar plates (diffusimetric method) with Staphylococcus aureus, Bacillus subtillis, Escherichia coli, and Salmonella typhi as test organisms. (Table-III)

Table -III Antibacterial Activity of Newly Synthesised Compounds, Zone of Inhibition (mm)

\begin{tabular}{|c|c|c|c|c|c|}
\hline Compd & $\mathrm{R}$ & S.aureus & B.subtillis & E.coli & S.typhi \\
\hline $3 a$ & 3'- $\mathrm{OCH}_{3}, 4^{\prime}-\mathrm{OH}$ & 13.0 & 11.0 & 12.0 & 9.0 \\
\hline $3 b$ & $4^{\prime}-\mathrm{CH}_{3}$ & 10.0 & 8.0 & 8.0 & 10.0 \\
\hline $3 c$ & $2^{\prime}-\mathrm{OH}$ & 11.0 & 12.0 & 8.0 & 10.0 \\
\hline $3 \mathrm{~d}$ & $4^{\prime}-\mathrm{OCH}_{3}$ & 12.0 & 10.0 & 9.0 & 12.0 \\
\hline $3 e$ & $3^{\prime}-\mathrm{NO}_{2}$ & 11.0 & 7.0 & 9.0 & 8.0 \\
\hline $3 f$ & $4^{\prime}-\mathrm{N}\left(\mathrm{CH}_{3}\right)_{2}$ & 9.0 & 10.0 & 11.0 & 11.0 \\
\hline $3 g$ & $2^{\prime}-\mathrm{NO}_{2}$ & 8.0 & 9.0 & 7.0 & 7.0 \\
\hline $3 \mathrm{~h}$ & $4^{\prime}-\mathrm{F}$ & 13.0 & 12.0 & 10.0 & 10.0 \\
\hline $3 \mathrm{i}$ & $2^{\prime}-\mathrm{OCH}_{3}$ & 8.0 & 10.0 & 10.0 & 12.0 \\
\hline $3 \mathrm{j}$ & $4^{\prime}-\mathrm{Cl}$ & 11.0 & 8.0 & 8.0 & 7.0 \\
\hline $4 a$ & $3^{\prime}-\mathrm{OCH}_{3}, 4^{\prime}-\mathrm{OH}$ & 12.0 & 7.0 & 11.0 & 9.0 \\
\hline $4 \mathrm{~b}$ & $4^{\prime}-\mathrm{CH}_{3}$ & 10.0 & 8.0 & 12.0 & 7.0 \\
\hline $4 \mathrm{c}$ & $2^{\prime}-\mathrm{OH}$ & 7.0 & 11.0 & 8.0 & 12.0 \\
\hline $4 d$ & $4^{\prime}-\mathrm{OCH}_{3}$ & 9.0 & 8.0 & 7.0 & 11.0 \\
\hline $4 \mathrm{e}$ & $3^{\prime}-\mathrm{NO}_{2}$ & 7.0 & 10.0 & 9.0 & 8.0 \\
\hline $4 \mathrm{f}$ & $4^{\prime}-\mathrm{N}\left(\mathrm{CH}_{3}\right)_{2}$ & 11.0 & 12.0 & 12.0 & 10.0 \\
\hline $4 \mathrm{~g}$ & $2^{\prime}-\mathrm{NO}_{2}$ & 8.0 & 10.0 & 9.0 & 12.0 \\
\hline
\end{tabular}




\begin{tabular}{cccccc}
\hline $4 \mathrm{~h}$ & $4{ }^{\prime}-\mathrm{F}$ & 13.0 & 9.0 & 10.0 & 7.0 \\
$4 \mathrm{i}$ & $2^{\prime}-\mathrm{OCH}_{3}$ & 10.0 & 12.0 & 7.0 & 10.0 \\
$4 \mathrm{j}$ & $4^{\prime}-\mathrm{Cl}$ & 9.0 & 7.0 & 10.0 & 9.0 \\
\hline
\end{tabular}

\section{Acknowledgement}

The authors are thankful to the South Gujarat University, Surat, INDIA for providing research facilities and CDRI, Lucknow, INDIA for providing ${ }^{1} \mathrm{H}$ NMR spectra and elemental analysis.

\section{References}

1 Sarvanan J, Mohan S and Manjunath K S, Indian J Heterocycl Chem, 1998, 8(1), 55.

2 Reddy P S N and Vasantha T V, Indian J Chem, 1999, 38B, 40.

3 Kumar A, Tyagi R, Bansal E, Verma R S, Saxena K K and Srivastava V K, Indian Drugs, 1998, 35(5), 261.

4 Deetz M J, Malerich J P, Beatty A M and Smith B D, Tetrahedron Lett, 2001, 42, 1851.

5 Ishida S, Matsuda A and Kawamura A, Chemotherapy, 1960, 8, 146; Chem Abstr, 1960, 54, 22844c.

6 Mehta K J, Patel V S and Parikh A R, J Indian Chem Soc, 1978, 50, 241.

$7 \quad$ Mudaliar V and Joshi V, Indian J Chem, 1995, 34B, 456.

8 Hishmat O H, El-Diwani H I and Melek F R, Indian J Chem, 1996, 35B, 30.

$9 \quad$ Karale B K and Gill C H, Indian J Chem, 2002, 41B, 1957.

10 Reddy V M and Sarma Rama G V S, Indian J Heterocycl Chem, 1993, 3, 111.

11 Shingare M S, Karale B K, Gill C H, Gange K N and Bachute M T, Indian J Heterocycl Chem, 1999, 9, 153.

12 Preeti Rani, Srivastava V K and Ashok Kumar, Ind J Chem, 2002, 41B, 2642. 


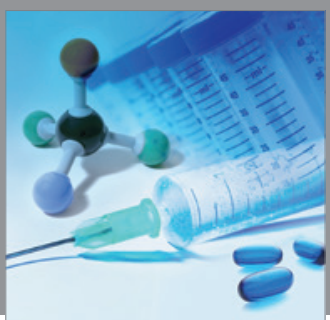

International Journal of

Medicinal Chemistry

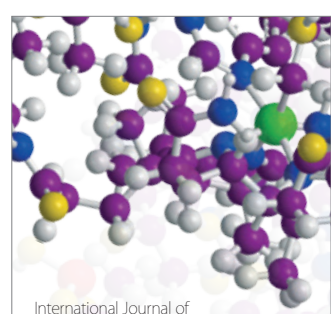

Carbohydrate Chemistry

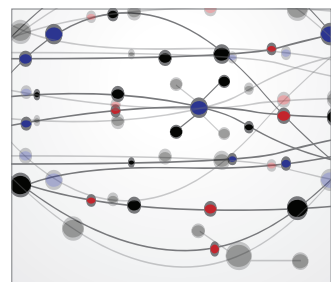

The Scientific World Journal
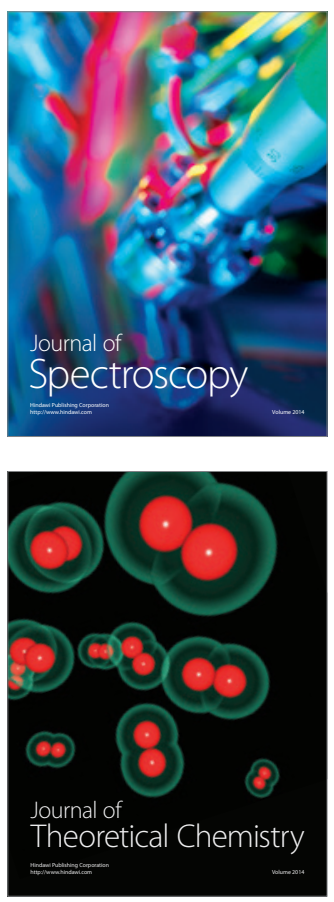
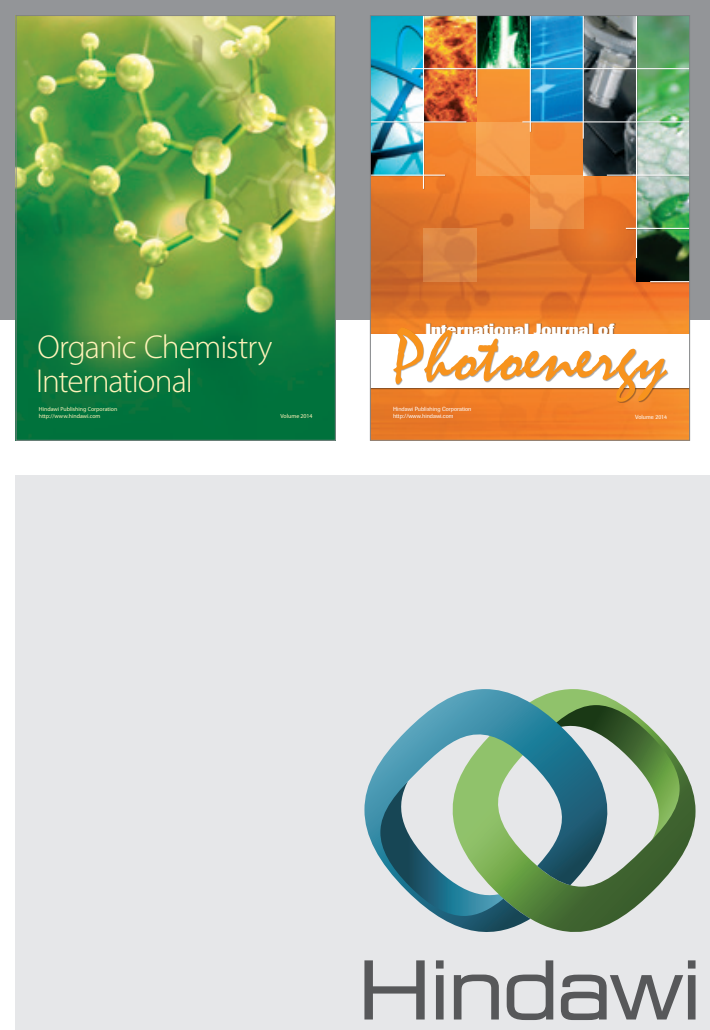

Submit your manuscripts at

http://www.hindawi.com
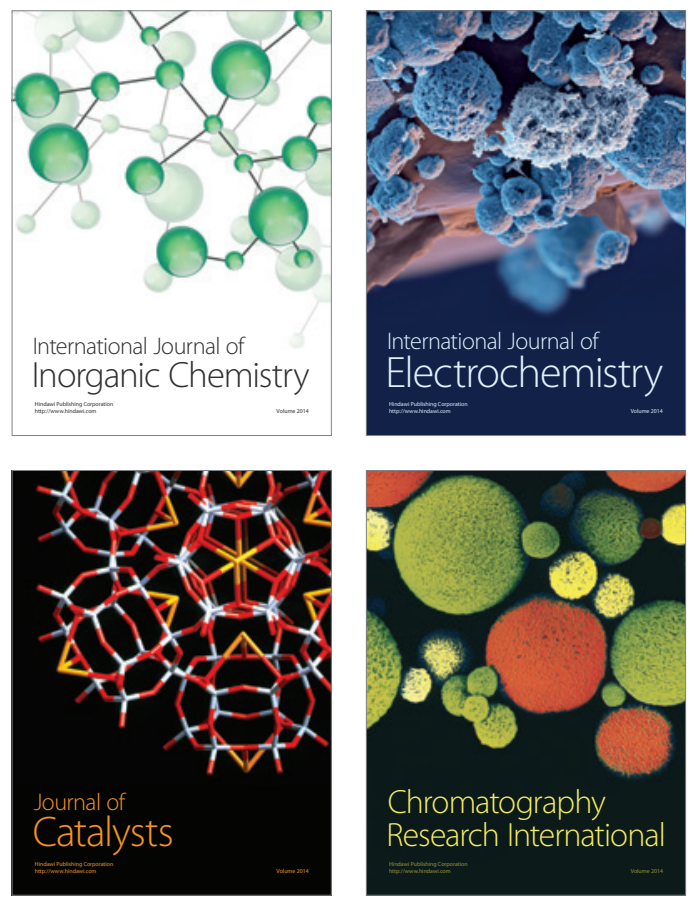
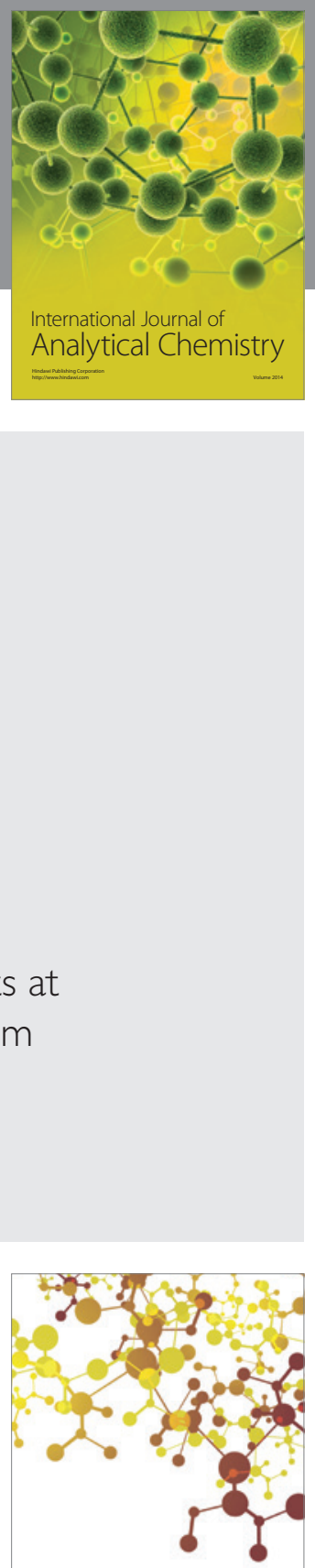

Journal of

Applied Chemistry
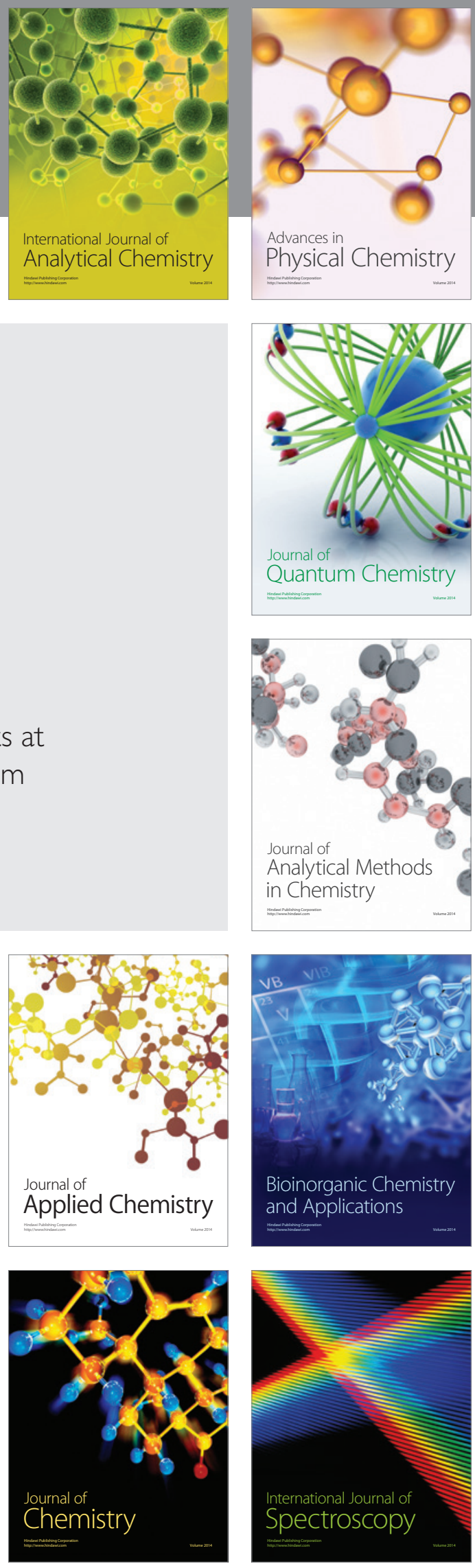